\title{
Retroperitoneal Hemorrhage, CTCAE 5.0
}

National Cancer Institute

\section{Source}

National Cancer Institute. Retroperitoneal Hemorrhage, CT CAE 5.0. NCI Thesaurus.

Code C146632.

A disorder characterized by bleeding from the retroperitoneal area. 